\title{
STUDY OF MICROORGANISMS WITHIN THE HOUSING ENVIRONMENT
}

\author{
Abdel Aziz, Heba, K. A. ${ }^{(1)}$; Howaihy, M. A..$^{(1)}$ and Arafa, Rawhia, A. ${ }^{(2)}$ \\ 1) Department of Basic Science, Institute of Environmental Studies \& Research, \\ Ain Shams University 2) Department of Botany and Microbiology, Faculty of \\ Science, Girl Branch, AL-Azhar University
}

\begin{abstract}
Bacteria are found virtually in every environment. High levels of indoor bacteria concentration is an indication of high occupancy rate, poor ventilation, or poor building maintenance. We study airborne Bacteria in Indoor Environments in two residences areas covering summer, winter season to study the climate effect (temperature) also covering high level and low level population to study the quality of life (good aeration, ventilation and variation of occupation area per citizens). We examine the presence of airborne Bacteria in Indoor Environments and colony forming unit for each case and we found that Exiguobacterium $\mathrm{sp}$ was the most frequent bacteria (37.5\%) in high level population during the Summer climate, Bacillus sp was the most frequent bacteria $(26.7 \%)$ in high level population during the Winter climate, Brachybacterium sp was the most frequent bacteria (42.4\%) during the summer climate and kytococcus sp was the most frequent bacteria (32.2\%) in low level population during the winter climate. We found a significant increase in colony forming unit among low level residence population during summer with Minimum, Maximum, Mean value 17.5, 25, $21.69 \mathrm{CFU} / \mathrm{m} 3$ respectively than among High level residence population in summer with Minimum, Maximum, Mean value $7.92,14.58,10.97 \mathrm{CFU} / \mathrm{m} 3$ respectively with $\mathrm{p}$-value $<0.001$ which indication the negative effect of high occupancy rate, poor ventilation on quality of life and a significant increase in colony forming unit in high level residence population in summer with Minimum, Maximum, Mean value 7.92, 14.58, 10.69 $\mathrm{CFU} / \mathrm{m} 3$ respectively than in high level residence population in winter with Minimum, Maximum, Mean value $0.42,2.5,1.39 \mathrm{CFU} / \mathrm{m} 3$ respectively with $\mathrm{p}$ value $<0.001$ which indicate high level of airborne bacteria due to high temperature .
\end{abstract}


Keywords: Air, Microorganisms, airborne Bacteria, Seasons and residences level

\section{INTRODUCTION}

How safe is the air in your surrounding environment that you spend much of your time? Indoor environments are fundamental environmental factors capable of impacting health. Air quality of indoor environments is one of the main factors affecting health, wellbeing and productivity of people. One of the problems of indoor air quality is affected by the presence of microorganisms which include bacteria, moulds and viruses (Wamedo S et al., 2012) People spends $80 \%-90 \%$ of their time in indoors environments[Awad \& Farag 1999] breathing on average $14 \mathrm{~m} 3$ of air per day[Brochu et al., 1999]. these make people highly exposed to indoor air environments. In recent years there has been a growing interest of indoor microbe studies [WHO 2009]. The activity of people within the indoor environments is thought to be the principal factor contributing to the buildup and spread of airborne microbial contamination [Qian et al., 2012]. Particular activities like talking, sneezing, coughing, walking and washing can generate airborne biological particulate matter. A review made by WHO on the number of epidemiological studies showed that there is sufficient evidence for an association between indoor dampness-related factors and a wide range of effects on respiratory health, including asthma development, asthma exacerbation, current asthma, respiratory infections, upper respiratory tract symptoms(cough, wheeze and dyspnea)[WHO 2009]. Thus microbiological air quality is an important criterion that must be taken into account when indoor workplaces are designed to provide a safe environment. This study provides 
information on the concentration of microorganisms and describes bacterial loads for different seasonal climate changes. Moreover, the impact of environmental factors (population level variation) on their multiplication and growth in the indoor air.Thus, the microbial loads of the buildings were favored by the environmental conditions which enhance their development. And also it was stated by WHO that dampness situation has to be considered as the risk indicator for health risks of biological contaminants of indoor air [WHO 2009].

\section{AIM OF STUDY}

- To identify and classify bacteria in our homes

- To assess the microbiological, indoor air quality (IAQ) in our home

- To evaluate the possible effect of temperature variation on bacterial growth.

\section{MATERIAL AND METHOD}

We study airborne Bacteria in Indoor Environments in two groups according to socioeconomic level (low and high), They were studied during summer and winter season to study the climate effect (temperature). High level population represent very spacious newly built \& well designed houses.(all residents have high income and social strata) and low level population represent poor houses, each was a single room or more but didn't exceed three room all which were built at random. (These shelters are inhabited by poor low income expatriate population usually more than 3 individuals per room)

To evaluate the concentration of bacteria in the indoor environment, study sample were collected indoor from 60 homes the sample were taken between 10:00 AM \& 12:00 PM. 
Samples was carried out using settle plates sedimentation technique, open Petri-dishes containing different culture media was employed to collect samples. Isolates were identified according to standard methods (Rajash and Rattan 2008). The settle plate method using $9 \mathrm{~cm}$ diameter Petri dishes. The sampling height was $1 \mathrm{~m}$ above the floor and at the center of the room. Bacteria were collected on nutrient and blood agar. To obtain the appropriate surface density for counting and to determine the load with respect to time of exposure, the sampling times were set at $60 \mathrm{~min}$. After that the covers are replaced and plates were then incubated at $37^{\circ}$ for 48 hours after which the colony forming units (CFU) were counted.

Once colony forming units (CFU) were enumerated, $\mathrm{CFU} / \mathrm{m}^{3}$ was estimated using Koch sedimentation method according to Polish Standard PN 89/Z04008/08 (Bhatica L and Vishwakarma 2010)

$$
\mathrm{CFU} / \mathrm{M}^{\wedge} 3=\frac{\text { colonies on agar stripes }}{\text { sampling time in munites }} \times 25
$$

Bacterial colonies were initially characterized by morphology and microscopic examination and identified further by biochemical tests using "Biolgo Gen 111 microplate TM " test panel which provides standardized micromethod using 94 biochemical test.

Data were analyzed by computer program. Descriptive statistics including percentage, mean and standard deviation were used for describing the bacterial count. 


\section{STATISTICAL ANALYSIS}

All analysis was done using the statistical package for the social science (SPSS software version 22) on a personal computer.

\section{RESULTS}

We study airborne Bacteria in Indoor Environments in four residence areas covering summer, winter season to study the climate effect (temperature)

Table (1): Colony count and identification of bacterial samples(Air borne Bacteria) in summer season high level population.

\begin{tabular}{|c|c|c|c|}
\hline $\begin{array}{c}\text { Sample } \\
\text { number }\end{array}$ & organism ID & $\begin{array}{c}\text { No } \\
\text { of Colony }\end{array}$ & CFUlm3 \\
\hline \hline \multirow{2}{*}{1} & Exiguobacterium aurantiacum & 24 & 10 \\
\cline { 2 - 4 } & Dietzia maris & 24 & 10 \\
\hline \multirow{3}{*}{2} & Exiguobacterium aurantiacum & 27 & 11.25 \\
\cline { 2 - 4 } & Dietzia maris & 28 & 11.67 \\
\cline { 2 - 4 } & Bacillus & 30 & 12.5 \\
\hline \multirow{3}{*}{3} & Exiguobacterium aurantiacum & 23 & 9.58 \\
\cline { 2 - 4 } & Dietzia maris & 24 & 10 \\
\cline { 2 - 4 } & Staphylo coccus arlettae & 24 & 10 \\
\hline \multirow{3}{*}{4} & Exiguobacterium aurantiacum & 20 & 8.33 \\
\cline { 2 - 4 } & Dietzia maris & 34 & 14.17 \\
\cline { 2 - 4 } & Staphylo coccus arlettae & 21 & 12.92 \\
\hline \multirow{2}{*}{5} & Exiguobacterium aurantiacum & 31 & 12.5 \\
\cline { 2 - 4 } & Dietzia maris & 30 & 12.92 \\
\hline \multirow{2}{*}{6} & Exiguobacterium aurantiacum & 30 & 31 \\
\cline { 2 - 4 } & Dietzia maris & \multicolumn{2}{|c||}{} \\
\hline
\end{tabular}


J. Environ. Sci.

Institute of Environmental Studies and Research - Ain Shams University

Cont.Table(1): Colony count and identification of bacterial samples(Air borne Bacteria) in summer season high level population.

\begin{tabular}{|c|c|c|c|}
\hline $\begin{array}{l}\text { sample } \\
\text { number }\end{array}$ & organism ID & No f Colony & CFUIm3 \\
\hline \multirow{2}{*}{7} & Exiguobacterium aurantiacum & 25 & 10.42 \\
\hline & Paenibacillus ginsengarvi & 27 & 11.25 \\
\hline \multirow{3}{*}{8} & Exiguobacterium aurantiacum & 30 & 12.5 \\
\hline & Dietzia maris & 35 & 14.58 \\
\hline & Bacillus & 32 & 13.33 \\
\hline \multirow{3}{*}{9} & Exiguobacterium aurantiacum & 22 & 9.17 \\
\hline & Dietzia maris & 25 & 10.42 \\
\hline & Staphylo coccus arlettae & 20 & 8.33 \\
\hline \multirow{3}{*}{10} & Exiguobacterium aurantiacum & 29 & 12.08 \\
\hline & Dietzia maris & 22 & 9.17 \\
\hline & Bacillus & 32 & 13.33 \\
\hline \multirow{3}{*}{11} & Exiguobacterium aurantiacum & 25 & 10.42 \\
\hline & Dietzia maris & 31 & 12.92 \\
\hline & Paenibacillus ginsengarvi & 30 & 12.5 \\
\hline \multirow{2}{*}{12} & Exiguobacterium aurantiacum & 22 & 9.17 \\
\hline & Dietzia maris & 25 & 10.42 \\
\hline \multirow{3}{*}{13} & Exiguobacterium aurantiacum & 24 & 10 \\
\hline & Dietzia maris & 20 & 8.33 \\
\hline & Staphylo coccus arlettae & 23 & 9.58 \\
\hline \multirow{3}{*}{14} & Exiguobacterium aurantiacum & 30 & 12.5 \\
\hline & Dietzia maris & 19 & 7.92 \\
\hline & Bacillus & 28 & 11.67 \\
\hline \multirow{3}{*}{15} & Exiguobacterium aurantiacum & 23 & 9.58 \\
\hline & Dietzia maris & 32 & 13.33 \\
\hline & Paenibacillus ginsengarvi & 24 & 10 \\
\hline
\end{tabular}


Table (2): Colony count and identification of bacterial samples (Air borne Bacteria) in winter season high level population.

\begin{tabular}{|c|c|c|c|}
\hline sample number & organism ID & NO OF COLONY & CFUlm3 \\
\hline \multirow{2}{*}{16} & Bacillus & 3 & 1.25 \\
\hline & Paenibacillus ginsengarvi & 1 & 0.417 \\
\hline \multirow{2}{*}{17} & Staphylo coccus arlettae & 3 & 1.25 \\
\hline & Dietzia maris & 1 & 0.42 \\
\hline \multirow{2}{*}{18} & Bacillus & 2 & 0.83 \\
\hline & Exiguobacterium aurantiacum & 3 & 1.25 \\
\hline \multirow{2}{*}{19} & Staphylo coccus arlettae & 3 & 1.25 \\
\hline & Dietzia maris & 4 & 1.67 \\
\hline \multirow{2}{*}{20} & Bacillus & 2 & 0.83 \\
\hline & Exiguobacterium aurantiacum & 5 & 2.08 \\
\hline \multirow{2}{*}{21} & Staphylo coccus arlettae & 5 & 2.08 \\
\hline & Dietzia maris & 3 & 1.25 \\
\hline \multirow{2}{*}{22} & Bacillus & 6 & 2.5 \\
\hline & Paenibacillus ginsengarvi & 2 & 0.83 \\
\hline \multirow{2}{*}{23} & Bacillus & 4 & 1.67 \\
\hline & Dietzia maris & 5 & 2.08 \\
\hline \multirow{2}{*}{24} & Staphylo coccus arlettae & 4 & 1.67 \\
\hline & Paenibacillus ginsengarvi & 3 & 1.25 \\
\hline \multirow{2}{*}{25} & Staphylo coccus arlettae & 3 & 1.25 \\
\hline & Exiguobacterium aurantiacum & 3 & 1.25 \\
\hline \multirow{2}{*}{26} & Bacillus & 2 & 0.83 \\
\hline & Exiguobacterium aurantiacum & 2 & 0.83 \\
\hline \multirow{2}{*}{27} & Staphylo coccus arlettae & 4 & 1.67 \\
\hline & Dietzia maris & 5 & 2.08 \\
\hline \multirow{2}{*}{28} & Bacillus & 6 & 2.5 \\
\hline & Exiguobacterium aurantiacum & 3 & 1.25 \\
\hline \multirow{2}{*}{29} & Staphylo coccus arlettae & 2 & 0.83 \\
\hline & Dietzia maris & 3 & 1.25 \\
\hline \multirow{2}{*}{30} & Bacillus & 6 & 2.5 \\
\hline & Exiguobacterium aurantiacum & 3 & 1.25 \\
\hline
\end{tabular}


J. Environ. Sci.

Institute of Environmental Studies and Research - Ain Shams University

Table(3): Colony count and identification of bacterial samples (Air borne Bacteria) in summer season low level population.

\begin{tabular}{|c|c|c|c|}
\hline $\begin{array}{c}\text { sample } \\
\text { number }\end{array}$ & organism ID & $\begin{array}{c}\text { No } \\
\text { of Colony }\end{array}$ & $\begin{array}{c}\text { CFUlm } \\
3\end{array}$ \\
\hline \multirow{2}{*}{31} & Brachy bacterium conglomeratum & 54 & 22.5 \\
\hline & Bacillus & 56 & 23.33 \\
\hline \multirow{2}{*}{32} & Brachy bacterium conglomeratum & 54 & 22.50 \\
\hline & Staphylo coccus arlettae & 49 & 20.42 \\
\hline \multirow{2}{*}{33} & Brachy bacterium conglomeratum & 52 & 21.67 \\
\hline & Bacillus & 44 & 18.33 \\
\hline \multirow{2}{*}{34} & Brachy bacterium conglomeratum & 52 & 21.67 \\
\hline & kytococcus aerolatus & 54 & 22.50 \\
\hline 35 & Staphylo coccus arlettae & $\varepsilon \varepsilon$ & $1 \wedge, \mu r$ \\
\hline \multirow{2}{*}{36} & Brachy bacterium conglomeratum & 42 & 17.5 \\
\hline & kytococcus aerolatus & 48 & 20.00 \\
\hline \multirow{3}{*}{37} & Brachy bacterium conglomeratum & 50 & 20.83 \\
\hline & kytococcus aerolatus & 50 & 20.83 \\
\hline & Bacillus & 55 & 22.92 \\
\hline \multirow{2}{*}{38} & Brachy bacterium conglomeratum & 52 & 21.67 \\
\hline & kytococcus aerolatus & 52 & 21.67 \\
\hline \multirow{2}{*}{39} & Brachy bacterium conglomeratum & 55 & 22.92 \\
\hline & Staphylo coccus arlettae & 56 & 23.33 \\
\hline \multirow{2}{*}{40} & Bacillus & 52 & 21.67 \\
\hline & Brachy bacterium conglomeratum & 60 & 25.00 \\
\hline \multirow{3}{*}{41} & Brachy bacterium conglomeratum & 55 & 22.90 \\
\hline & kytococcus aerolatus & 54 & 22.5 \\
\hline & Staphylo coccus arlettae & 55 & 22.90 \\
\hline \multirow{3}{*}{42} & Brachy bacterium conglomeratum & 59 & 24.6 \\
\hline & kytococcus aerolatus & 52 & 21.67 \\
\hline & Bacillus & 50 & 20.83 \\
\hline \multirow{3}{*}{43} & Brachy bacterium conglomeratum & 48 & 20.00 \\
\hline & Staphylo coccus arlettae & 48 & 20 \\
\hline & kytococcus aerolatus & 54 & 22.5 \\
\hline \multirow{2}{*}{44} & Brachy bacterium conglomeratum & 52 & 21.67 \\
\hline & kytococcus aerolatus & 51 & 21.25 \\
\hline \multirow{3}{*}{45} & Brachy bacterium conglomeratum & 52 & 21.70 \\
\hline & kytococcus aerolatus & 56 & 23.30 \\
\hline & Staphylo coccus arlettae & 53 & 22.1 \\
\hline
\end{tabular}


Table (4): Colony count and identification of bacterial samples(Air borne Bacteria) in winter season low level population.

\begin{tabular}{|c|c|c|c|}
\hline $\begin{array}{c}\text { sample } \\
\text { number }\end{array}$ & organism ID & $\begin{array}{c}\text { No } \\
\text { of Colony }\end{array}$ & CFUlm3 \\
\hline \multirow{2}{*}{46} & kytococcus aerolatus & 7 & 2.92 \\
\hline & Bacillus & 8 & 3.33 \\
\hline \multirow{2}{*}{47} & Brachy bacterium conglomeratum & 11 & 4.58 \\
\hline & Staphylo coccus arlettae & 10 & 4.17 \\
\hline \multirow{3}{*}{48} & Brachy bacterium conglomeratum & 9 & 3.75 \\
\hline & kytococcus aerolatus & 10 & 4.17 \\
\hline & Bacillus & 8 & 3.33 \\
\hline \multirow{2}{*}{49} & kytococcus aerolatus & 7 & 2.92 \\
\hline & Staphylo coccus arlettae & 8 & 3.33 \\
\hline \multirow{2}{*}{50} & Brachy bacterium conglomeratum & 7 & 2.92 \\
\hline & Staphylo coccus arlettae & 9 & 3.75 \\
\hline \multirow{3}{*}{51} & Brachy bacterium conglomeratum & 11 & 4.58 \\
\hline & kytococcus aerolatus & 12 & 5 \\
\hline & Bacillus & 15 & 6.25 \\
\hline \multirow{2}{*}{52} & kytococcus aerolatus & 15 & 6.25 \\
\hline & Bacillus & 10 & 4.17 \\
\hline \multirow{2}{*}{53} & Brachy bacterium conglomeratum & 15 & 6.25 \\
\hline & kytococcus aerolatus & 11 & 4.58 \\
\hline 54 & Staphylo coccus arlettae & 15 & 6.25 \\
\hline \multirow{2}{*}{55} & kytococcus aerolatus & 15 & 6.25 \\
\hline & Bacillus & 13 & 5.42 \\
\hline \multirow{2}{*}{56} & kytococcus aerolatus & 14 & 5.83 \\
\hline & Staphylo coccus arlettae & 14 & 5.83 \\
\hline \multirow{2}{*}{57} & kytococcus aerolatus & 13 & 5.42 \\
\hline & Staphylo coccus arlettae & 12 & 5 \\
\hline \multirow{2}{*}{58} & kytococcus aerolatus & 12 & 5 \\
\hline & Bacillus & 12 & 5 \\
\hline \multirow{2}{*}{59} & Brachy bacterium conglomeratum & 11 & 4.58 \\
\hline & Bacillus & 10 & 4.17 \\
\hline \multirow{2}{*}{60} & Brachy bacterium conglomeratum & 11 & 4.58 \\
\hline & Staphylo coccus arlettae & 12 & 5 \\
\hline
\end{tabular}


Table (5): Frequencies of airborne bacteria in High level residence during Summer season.

\begin{tabular}{|c|c|c|c|}
\hline & Organism identification & Frequency & Percent \% \\
\hline \hline 1 & Bacillus & 4 & $10.0 \%$ \\
\hline 2 & Dietzia maris & 14 & $35.0 \%$ \\
\hline 3 & Exiguobacterium aurantiacum & 15 & $37.5 \%$ \\
\hline 4 & Paenibacillus ginsengarvi & 3 & $7.5 \%$ \\
\hline 5 & Staphylococcus arlettae & 4 & $10.0 \%$ \\
\hline
\end{tabular}

Table (6): Frequencies of airborne bacteria in High level residence during winter season.

\begin{tabular}{|c|c|c|c|}
\hline & Organism identification & Frequency & Percent \\
\hline \hline 1 & Bacillus & 8 & $26.7 \%$ \\
\hline 2 & Dietzia maris & 6 & $20.0 \%$ \\
\hline 3 & Exiguobacterium aurantiacum & 6 & $20.0 \%$ \\
\hline 4 & Paenibacillus ginsengarvi & 3 & $10.0 \%$ \\
\hline 5 & Staphylococcus arlettae & 7 & $23.3 \%$ \\
\hline
\end{tabular}

Table (7): Frequencies of airborne bacteria in Low level residence during Summer season.

\begin{tabular}{|c|c|c|c|}
\hline & Organism identification & Frequency & Percent \\
\hline \hline 1 & Bacillus & 4 & $12.1 \%$ \\
\hline 2 & Brachybacterium conglomeratum & 14 & $42.4 \%$ \\
\hline 3 & kytococcus aerolatus & 9 & $27.3 \%$ \\
\hline 4 & Staphylococcus & 6 & $18.2 \%$ \\
\hline & Total & 65 & $100.0 \%$ \\
\hline
\end{tabular}

Table(8): Frequencies of airborne bacteria in Low level residence during Winter season.

\begin{tabular}{|c|c|c|c|}
\hline & Organism identification & Frequency & Percent \\
\hline \hline 1 & Bacillus & 7 & $22.6 \%$ \\
\hline 2 & Brachybacterium conglomeratum & 7 & $22.6 \%$ \\
\hline 3 & kytococcus aerolatus & 10 & $32.2 \%$ \\
\hline 4 & Staphylococcus & 7 & $22.6 \%$ \\
\hline \multicolumn{3}{|c}{} \\
\hline
\end{tabular}


Table (9): Comparison of colony forming units of airborne bacteria during two seasons

\begin{tabular}{|c|c|c|c|c|}
\hline & \multicolumn{4}{|c|}{ CFUlm $^{\text {s }}$} \\
\hline Variable & Minimum & Maximum & Mean & Std. Deviation \\
\hline \hline Summer high level & 7.92 & 14.58 & 10.9687 & 1.82083 \\
\hline Winter high level & .42 & 2.50 & 1.3889 & .59263 \\
\hline Summer low level & 17.50 & 25.00 & 21.6913 & 1.64674 \\
\hline Winter low level & 2.92 & 6.25 & 4.6640 & 1.06729 \\
\hline
\end{tabular}

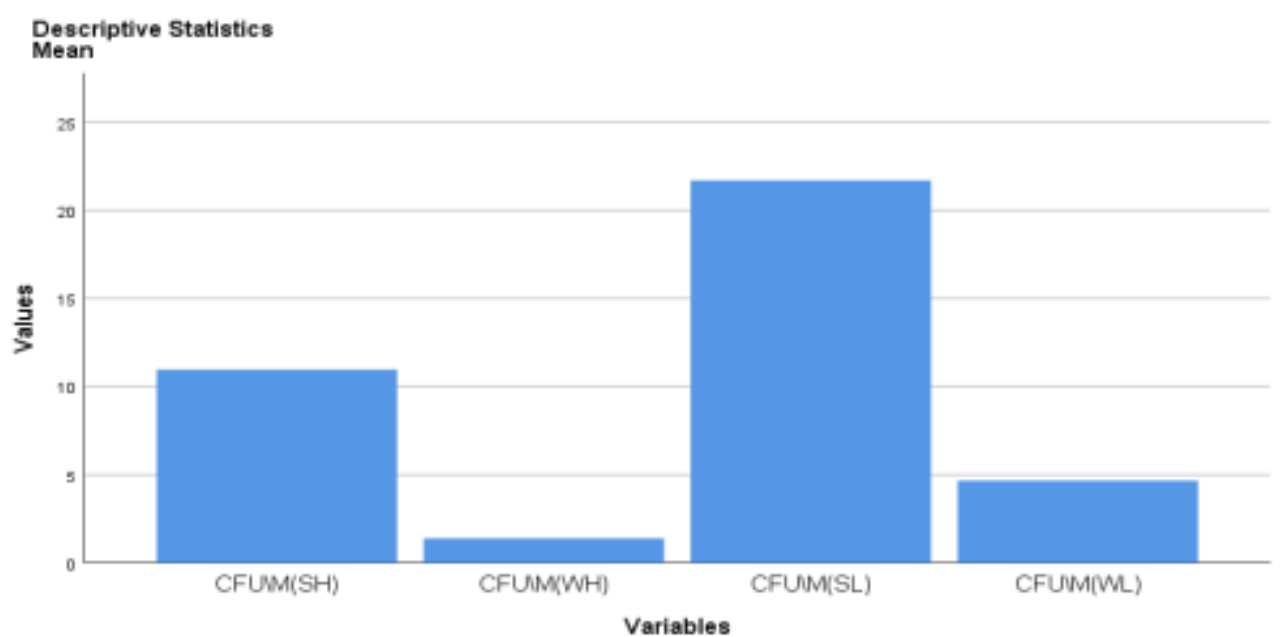

Figure (1): Comparison of colony forming units of airborne bacteria in the two levels.

* SH: high level population in summer, * WH: high level population in winter, *SL: low level population in summer, *WL: low level population in winter.

Table (10): Comparison of colony forming units of airborne bacteria among low level residence during two seasons.

\begin{tabular}{|c|c|c|c|c|l|}
\hline \multirow{2}{*}{ Season } & \multicolumn{3}{|c|}{ CFUlm3 } & $\begin{array}{c}\text { Std. } \\
\text { Deviation }\end{array}$ & p-value \\
\cline { 2 - 5 } & Minimum & Maximum & Mean & \\
\hline \hline Summer season & 17.50 & 25.00 & 21.6913 & 1.64674 & \multirow{2}{*}{$\mathrm{P}<0.001$} \\
\hline Winter season & 2.92 & 6.25 & 4.6640 & 1.06729 & \\
\hline
\end{tabular}


J. Environ. Sci.

Institute of Environmental Studies and Research - Ain Shams University

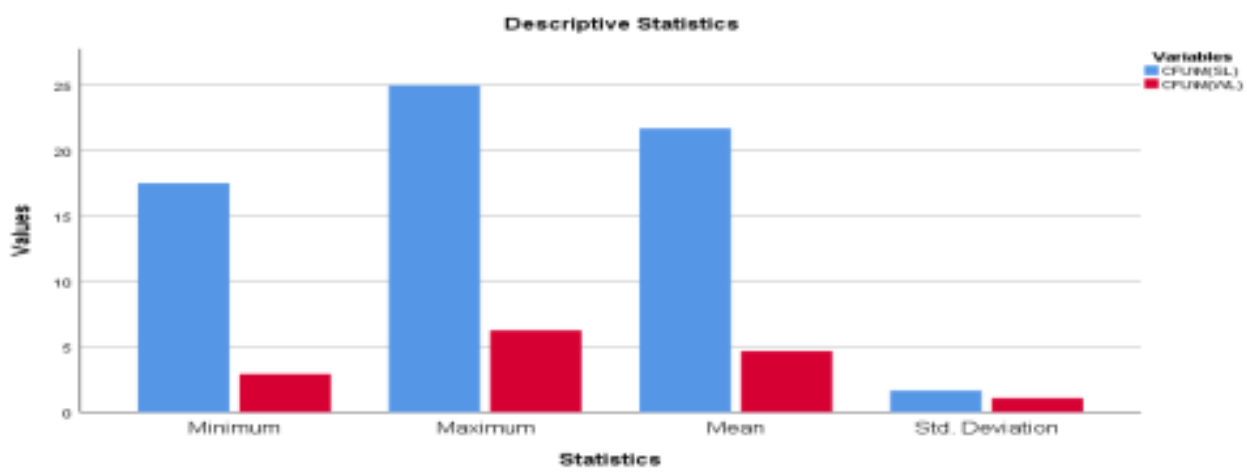

Figure (2): Comparison of colony forming units of airborne bacteria among low level residence during two seasons to study climate effect in airborne bacteria.

*SL : low level population in summer, *WL : low level population in winter

Table (11): Comparison of colony forming units of airborne bacteria among High level residence during two seasons .

\begin{tabular}{|c|c|c|c|c|c|}
\hline \multirow{2}{*}{ Season } & \multicolumn{3}{|c|}{ CFUlm $^{\text {S }}$} & $\begin{array}{c}\text { Std. } \\
\text { Deviation }\end{array}$ & p-value \\
\cline { 2 - 5 } & Minimum & Maximum & Mean & \\
\hline Winter season & 0.42 & 2.50 & 1.3889 & 0.59263 & \\
\hline Summer season & 7.92 & 14.58 & 10.9687 & 1.82083 & $\mathrm{P}<0.001$ \\
\hline
\end{tabular}

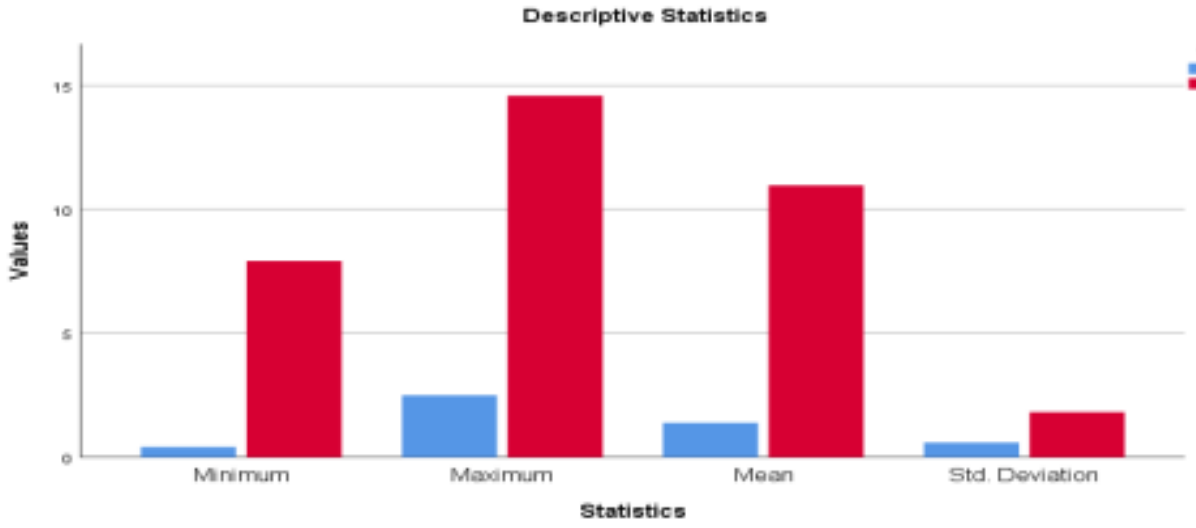

Figure (3): Comparison of colony forming units of airborne bacteria among

High level residence during two seasons.

* SH : high level population in summer, * WH : high level population in winter 
Table (12): Comparison of colony forming units of airborne bacteria among high and low level residence during one summer season

\begin{tabular}{|c|c|c|c|c|c|}
\hline $\begin{array}{c}\text { Socioeconomic } \\
\text { level }\end{array}$ & \multicolumn{3}{|c|}{ CFU/m3 } & $\begin{array}{c}\text { Std. } \\
\text { Deviation }\end{array}$ & p-value \\
\cline { 2 - 5 } & Minimum & Maximum & Mean & 1.64674 & \\
\hline \hline low level & 17.50 & 25.00 & 21.6913 & 1.82083 & $\mathrm{P}<0.001$ \\
\hline High level & 7.92 & 14.58 & 10.9687 & 1.8200 \\
\hline
\end{tabular}

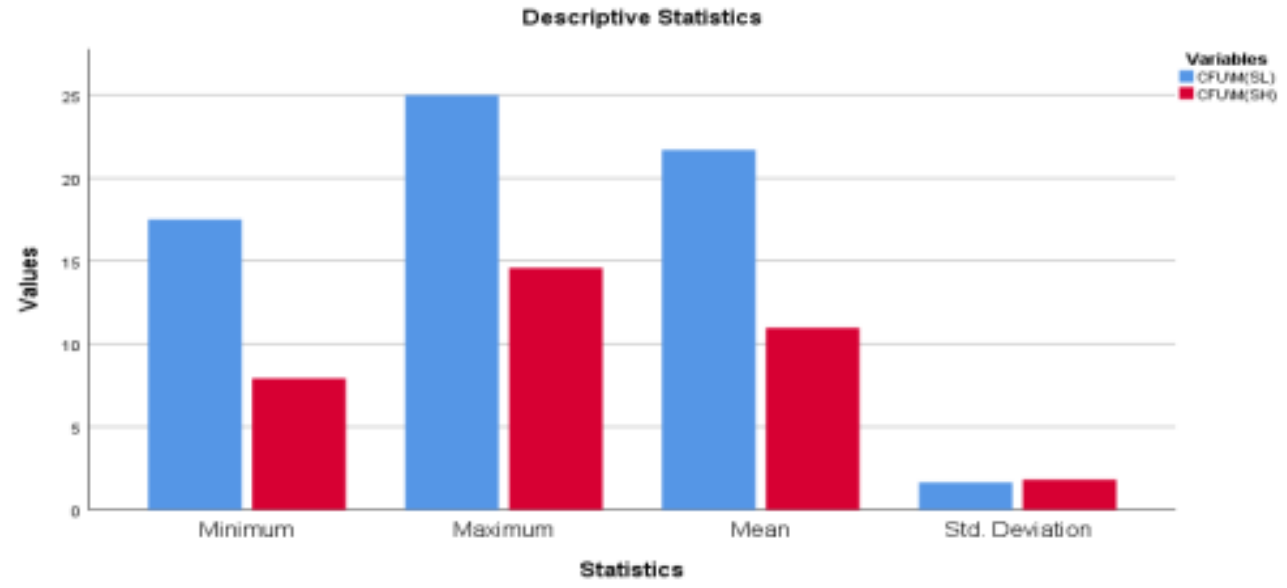

Figure (4): Comparison of colony forming units of airborne bacteria between high level residence and low level population during summer .

* SH : high level population in summer, *SL : low level population in summer,

Table (13): Comparison of colony forming units of airborne bacteria among high and low level residence during winter season

\begin{tabular}{|c|c|c|c|c|c||}
\hline $\begin{array}{c}\text { Socioeconomic } \\
\text { level }\end{array}$ & \multicolumn{3}{|c|}{ CFU/m3 } & $\begin{array}{c}\text { Std. } \\
\text { Deviation }\end{array}$ & p-value \\
\cline { 2 - 5 } & Minimum & Maximum & Mean & \\
\hline High level & .42 & 2.50 & 1.3889 & 0.59263 & \\
\hline low level & 2.92 & 6.25 & 4.6640 & 1.06729 & $\mathrm{P}<0.001$ \\
\hline
\end{tabular}


J. Environ. Sci.

Institute of Environmental Studies and Research - Ain Shams University

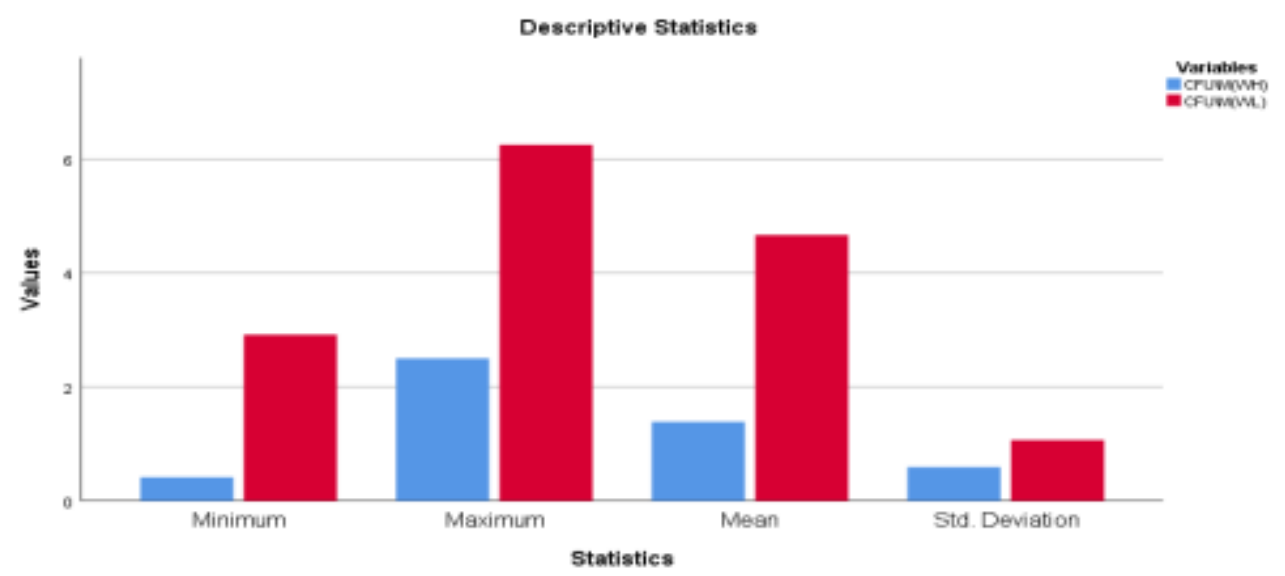

Figure (5): Comparison of colony forming units of airborne bacteria between high level residence and low level population during winter.

* WH : high level population in winter, *WL : low level population in winter

\section{DISCUSSION}

A review made by WHO on the number of epidemiological studies showed that, there is sufficient evidence for an association between indoor dampnessrelated factors and a wide range of effects on respiratory health, including asthma development, asthma exacerbation, current asthma, respiratory infections, upper respiratory tract symptoms, cough, wheeze and dyspnoea (WHO 2009) The activity of people and equipment within the indoor environments is thought to be the principal factor contributing to the buildup and spread of airborne microbial contamination(Hospodsky et al., 2012) Moreover, the environmental factors mainly include temperature, humidity, air exchange rate, air movement, building structures and location, poor design, ventilation system as well as interior or redesign which enhance microorganism's growth and multiplication in the indoor atmosphere.( Wamedo et al., 2012). 50 
Tables 1, 2, 3 and 4 show colony count and identification of airborne bacteria in the two residence areas. Statistical analysis of these four tables shows that Exiguobacterium sp was the most frequent bacteria $(37.5 \%)$ followed by Dietzia sp (35\%), staphylococcus sp (10\%), Bacillus sp (10\%) and the less frequent Paenibacillus sp 7.5\% among high level population during the Summer season (table 5). Bacillus sp was the most frequent bacteria (26.7\%) followed by staphylococcus sp (23.3\%), Exiguobacterium sp(20\%), Dietzia sp (20\%) and the less frequent Paenibacillus $\operatorname{sp}(10 \%)$ among high level population during the Winter season (table 6). Brachybacterium $\mathrm{sp}$ was the most frequent bacteria (42.4\%) followed by kytococcus spv(27.3\%), staphylococcus sp (18.2\%) and the less frequent Bacillus sp (12.1\% )in low level population in the summer climate(table 7).kytococcus sp was the most frequent bacteria (32.2\%) followed by Brachybacterium sp, staphylococcus sp, Bacillus sp (22.6\%)in low level population in the winter climate(table 8).

Colony forming unit was maximum in summer season low level population (table 9) which indicate high level of airborne bacteria due to high temperature and low quality of life as shown in Fig 1.

A significant increase in colony forming unit in low level residence population in summer with Minimum, Maximum, Mean value 17.5, 25, 21.69 $\mathrm{CFU} / \mathrm{m} 3$ respectively than in low level residence population in winter with Minimum, Maximum, Mean value 2.92, 6.25, $4.66 \mathrm{CFU} / \mathrm{m} 3$ respectively with p-value $<0.001$ (table 10) which indicate high level of airborne bacteria due to high temperature as shown in Fig 2.

A significant increase in colony forming unit in high level residence population in summer with Minimum, Maximum, Mean value 7.92, 14.58, 10.69 
$\mathrm{CFU} / \mathrm{m} 3$ respectively than in high level residence population in winter with Minimum, Maximum, Mean value $0.42,2.5,1.39 \mathrm{CFU} / \mathrm{m} 3$ respectively with $\mathrm{p}$ value $<0.001$ (table 11) which indicate high level of airborne bacteria due to change in temperature as shown in Fig 3.

A significant increase in colony forming unit in low level residence population in summer with Minimum, Maximum, Mean value 17.5, 25, 21.69 $\mathrm{CFU} / \mathrm{m} 3$ respectively than in high level residence population during summer with Minimum, Maximum, Mean value 7.92, 14.58, 10.97 CFU/m3 respectively with p-value $<0.001$ (table 12) which indicate high level of airborne bacteria due to low ventilation and cleaning facilities as shown in Fig 4.

A significant increase in colony forming unit in low level residence population during winter with Minimum, Maximum, Mean value 2.92, 6.25, 4.66 CFU/m3 respectively than in High level residence population in winter with Minimum, Maximum, Mean value 0.42, 2.5, $1.39 \mathrm{CFU} / \mathrm{m} 3$ respectively with p-value $<0.001$ (table 13) which indicate high level of airborne bacteria due to low ventilation and cleaning facilities as shown in Fig 5.

The concentrations of bacteria measured in all sites were significantly different to each other (P-value $<0.001)$. These can be mainly explained by the variation of density of occupant during sampling time in low level population as well as the variation of ventilation conditions and climate changes 


\section{CONCLUSION\& RECOMMENDATION}

According to statistical study of samples in the two levels there were significant differences in bacterial growth which reflect the effect of socioeconomical quality of life and also reflects the effect of climate (temperature) change in bacterial growth in indoor environment. Thus:

1. Attention must be given to control environmental factors which favor the growth and multiplication of microbes in indoor environment.

2. Further studies must be done to asses health effect on human due to indoor air pollution by airborne bacteria.

3. Increasing health education to socioeconomic low level population.

\section{REFERENCES}

Awad AH and Farag SA (1999): An indoor bio-contaminants air quality. Int J Environ Health Res; 9: 313-319.

Bhatica L and Vishwakarma R(2010): Hospital Indoor Airborne Microflora in private and Government owned Hospitals in Sagar City, India. World J. Med. Sci. 5 (3): 65-70

Borrego S(2010): Guiamet P, de Saravia SG, Batistini P, Garcia M, Lavin P, et al. The quality of air at archives and the biodeterioration of photographs. Int Biodeterior Biodegradation. ;64:139-145.

Brochu P(2006): Ducré-Robitaille JF, Brodeur J. Physiological daily inhalation rates for free-living individuals aged 1 month to 96 years, using data from doubly labeled water measurements: a proposal for air quality criteria, standard calculations and health risk assessment. Human Ecol Risk Assess; 12: 675- 701.

Hospodsky D(2012): Qian J, Nazaroff WW, Yamamoto N, Bibby K, RismaniYazdi H, Human Occupancy as a Source of Indoor Airborne bacteria.PLoSONE7(4):e34867.

https://doi.org/10.1371/journal.pone.0034867 
Qian J(2012): Hospodsky D, Yamamoto N, Nazaroff WW, Peccia J. Sizeresolved emission rates of airborne bacteria and fungi in an occupied classroom. Indoor Air; 22: 339-351.

Rajash B(2008): Rattan LI. Essential of medical microbiology. 4th ed. New Delhi: Jayppe Brothers Medical Publishers;. pp. 415-439.

Wamedo SA(2012): Ede PN, Chuku A. Interaction between building design and indoor airborne microbial load in Nigeria. Asian J Biol Sci; 5: 183191.

World Health Organization(2009): WHO guidelines for indoor air quality: dampness and mould. Copenhagen, Denmark: World Health Organization.

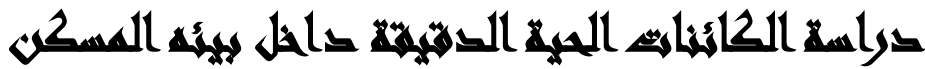

$[r]$

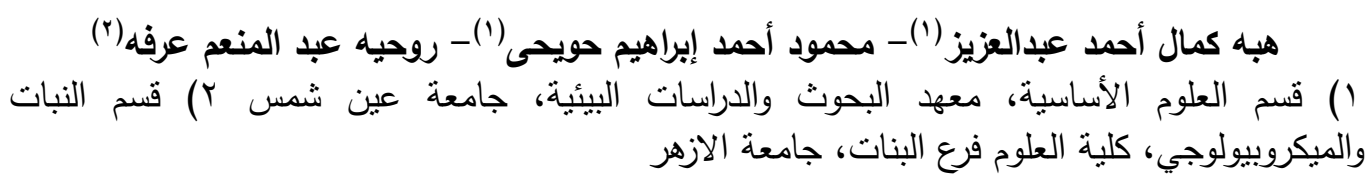

\section{المستخلص}

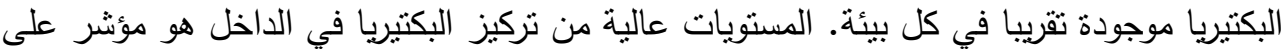

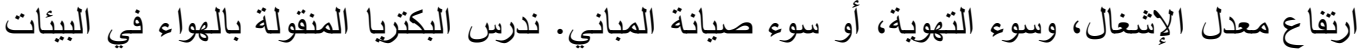

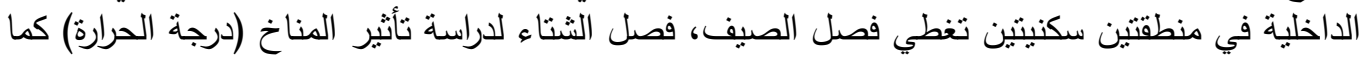

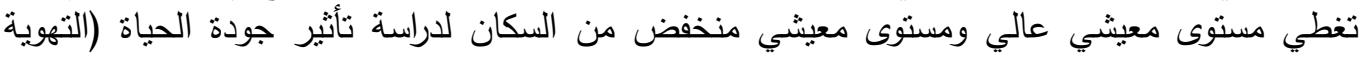
الجيدة والتهوية وتغيير المساحة المتاحة لكل مواطن) في التلوث بالبكتريا في الأماكن المغلقة. ندرس لتون

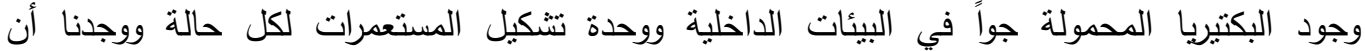
Heliobacterium sp

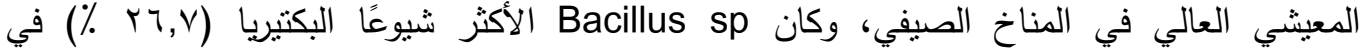

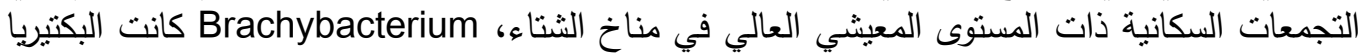

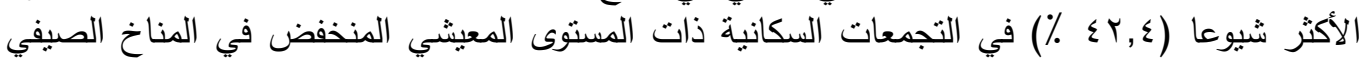

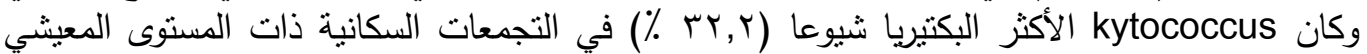
المنخفض في مناخ الثتاء. وجدنا زيادة ملحوظة في وحدة نتكيل المستعمرات في التجمعات السكانية 
ذات المستوى المعيثي المنخفض في المناخ الصيفي مع الحد الأدنى، الحد الأقصى، القيمة المتوسطة

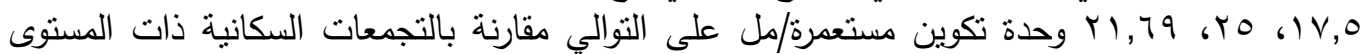

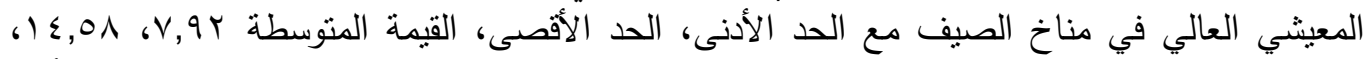

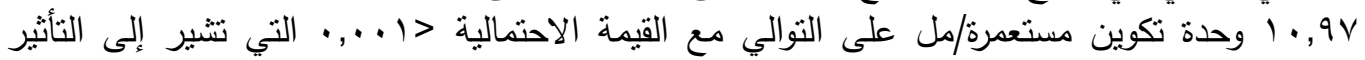

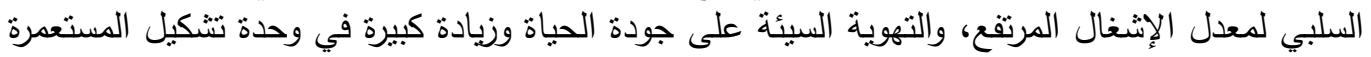

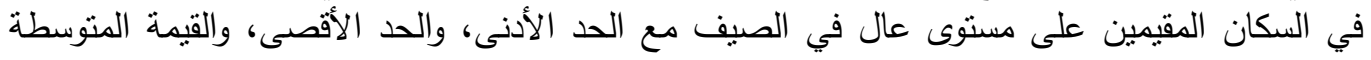

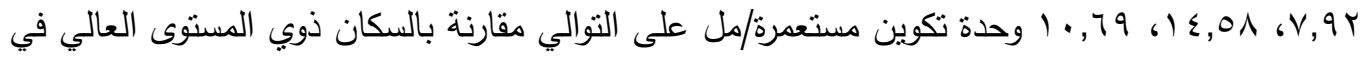

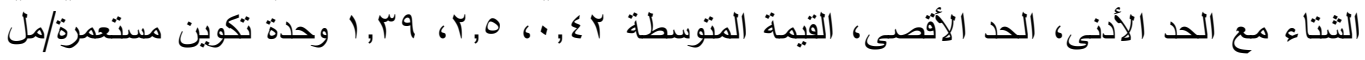

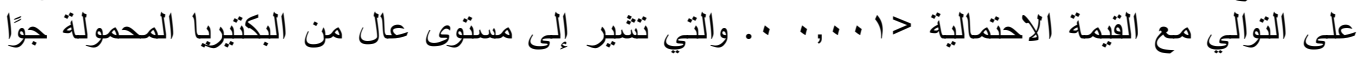

بسبب ارتفاع درجة الحرارة . 